\title{
Acerca de los usos y algunos abusos de lo cualitativo y de la etnografía
}

\author{
Lenín Calderón ${ }^{1}$
}

\section{A manera de introducción}

A pesar de la hegemonía positivista, puede considerarse que aún existe (por lo menos en buena parte de los centros e institutos de educación superior en Venezuela) lo que algunos han definido como el "boom cualitativo", en tanto cierta tendencia hacia la utilización y aplicación de procesos metodológicos "no convencionales" o que están fuera del "marco metodológico positivista", en donde, entre otros métodos, la etnografía se encuentra con unos niveles de popularidad sin precedentes.

Quizás resulte reiterativo, y sin intenciones de hacer un tratado al respecto, pero siempre será necesario señalar que desde hace ya un buen tiempo se viene reflexionando acerca de cómo se produce el conocimiento y acerca de la validez y pertinencia de las formas en las cuales éste se produce. Como se sabe, existe o ha existido un fuerte cuestionamiento a algunos de los principios y nociones del denominado "método científico", sobre todo a su aplicación y desarrollo en el ámbito de la investigación de los fenómenos sociales. Cuestionamientos que por cierto no solo provinieron o provienen de aquellos que desde las "ciencias duras" o naturales aún reniegan la posibilidad de producir conocimiento científico de lo social, sino también de aquellos que, a favor de conocer "científicamente" la realidad social, igual se oponen al trasplante del método científico a este ámbito.

Estos cuestionamientos, que a decir verdad no son ni remotamente recientes, si tomamos en cuenta, por ejemplo, las diferencias entre racionalismo y empirismo que se dieron entre Platón

$1 \quad$ Doctorado en Ciencias Humanas. Maestría en Antropología. Sociólogo. Docente investigador de la Universidad Nacional Experimental Rafael María Baralt (Venezuela). 
y Aristóteles, en donde se planteaba, por una parte, que la razón o la "acción" reflexiva de una idea es el fundamento del conocimiento $y$, por la otra, se afirmaba que el conocimiento tiene su origen en los hechos concretos. De esta manera, toda la tradición reflexiva epistemológica realizada desde entonces y hasta ahora, siempre ha apuntado a establecer las bondades y ventajas, pero también las debilidades y el agotamiento de los modelos tradicionales de aprehensión y comprensión de la realidad a lo largo de la historia.

En tal sentido, de los cuestionamientos y reflexiones más significativas, se pueden mencionar a las discusiones entre las llamadas "ciencias de la naturaleza y ciencias del espíritu" propuestas o iniciadas por Dilthey en la segunda mitad del siglo XVIII (Peñaranda, 2004), pasando por las ideas fundadas e inspiradas por el relativismo a principios del Siglo XX, y que de alguna manera hicieron repensar la validez universal de los postulados científicos, hasta llegar hoy a lo que se conoce como la "verdad como perspectiva" del posmodernismo, entre otras muchas. Los referidos planteamientos, son reflexiones sobre el cómo se produce o debe producirse el conocimiento, con las cuales se puede o no estar de acuerdo, pero que no pueden ser menospreciados u obviados como lo intentan hacer algunos de los más furibundos defensores del "cientificismo puro" y mucho menos reducirlas a una discusión dicotómica entre lo cuantitativo y lo cualitativo.

Con el inicio del siglo XX, se consolidó la idea de que la modernidad salvaría a la humanidad, basado en el principio de que la ciencia (razón tecno-científica) era la única manera válida y absoluta de abordar, percibir, incluso de relacionarnos socialmente y, dado el caso, de transformar la realidad. Sin embargo ello no ha sido necesariamente así. Gran parte de los grandes problemas que aquejan hoy a la humanidad se han desarrollado a la par de los grandes avances científicos y tecnológicos, entre otros aspectos precisamente, por las limitaciones que el enfoque racional-científico ha demostrado tener para entender de manera cabal lo social y todas las realidades que esta encierra. De tal manera que la pretensión de entender de la misma forma cómo se entiende al mundo natural a las relaciones humanas, sociales y/o culturales, como bien lo plantearan Durkhein, Comte, y 
tantos otros, es decir, únicamente en términos de relaciones causales o la de descubrir y establecer leyes universales, tiene una larga data de reflexión y por ello mismo de cuestionamientos.

Como quiera que sea y cualquiera sean las observaciones, coincidencias y discrepancias que se puedan tener contra la epistemología que indaga más allá del método científico (que no en pocos casos puede resultar verdaderamente especulativa en mi criterio), algo sobre lo cual se tenderá a estar de acuerdo (por lo menos) es acerca de ciertas dudas que generan algunos principios fundamentales de la ciencia, sobre todo cuando éstos principios se intentan aplicar dentro del ámbito de lo sociocultural.

Sin embargo, también vale la pena destacar que los cuestionamientos referidos no suponen siempre y necesariamente una negación y/o "satanización" a priori de los modelos tradicionales, o del positivismo en específico. Al respecto, suele suceder que quienes desde los enfoques "post-positivistas" se solazan en las debilidades del positivismo no hacen más que esconder en muchos casos el desconocimiento de los aportes y utilidad del mismo en el desarrollo de las disciplinas y ciencias de las cuales muchas veces forman parte.

De tal manera que, precisamente el "agotamiento" al cual me refiero apunta a la limitación, cuando no imposibilidad, del enfoque positivista para explicar todas y las distintas realidades de lo sociocultural de una manera que no deje cierta gama de sinsabores.

Este tipo de situación referido a la imposibilidad de un modelo de ofrecer nuevos enfoques y/o formas para la explicación de los fenómenos, ha sido lo que siempre ha impulsado la necesidad de indagar, de "experimentar" la utilización de otras perspectivas y/o enfoques que permitan, cada vez más, acercarse, profundizar en el entendimiento de las distintas realidades, que dicho sea de paso, es la forma cómo se ha desarrollado el conocimiento a lo largo de la historia humana, y es consustancial a la ciencia misma, y es en este contexto que surgen los llamados "nuevos modelos", paradigmas los llamó Thomas Kuhn (1971), que enmarcan distintas maneras de cómo aproximarse a la realidad social y humana, como es el caso de la etnografía. De allí su actual popularidad. 


\section{El asunto que me atiende}

El "boom cualitativo" y la popularidad de la etnografía ya era señalada, a finales del siglo recientemente pasado, por M. Hammersley y P. Atkinson (1993) quienes aseguraban que:

En los últimos años, entre los investigadores de varios campos ha crecido el interés; tanto teórico como práctico, por la etnografía. En gran medida, ello se debe a la desilusión provocada por los métodos cuantitativos, métodos que han detentado durante mucho tiempo una posición dominante en la mayor parte de las ciencias sociales (p. 1).

A estas alturas, en lo particular considero una quimera la discusión acerca de la distinción entre lo cuantitativo y lo cualitativo en tanto clasificaciones válidas para los tipos de investigación, e incluso aún más, cuando se utilizan dichas distinciones para determinar si una investigación es "buena o mala", pero no es mi intención aquí tratar el asunto.

Sin embargo, es importante señalar que de alguna manera entiendo que dichas distinciones contienen o pudieran contener el germen que, precisamente, impulsa la búsqueda de nuevas o mejores formas para producir conocimiento, esa es la virtud que puedo reconocer a tal distinción. Lo que me interesa destacar tiene que ver con un fenómeno que viene afectando a aquellos que asumimos la etnografía como una forma válida y necesaria para el entendimiento de los procesos socioculturales.

Desde la antropología, en específico F. Boas (1911), fue uno de los primeros que reconoció las limitaciones del método científico para la mejor explicación de los fenómenos socioculturales. Este planteamiento establece que ningún aspecto sociocultural puede ser explicado fuera del entendimiento y comprensión de su propio contexto que le dio vida. Es decir, sin la comprensión histórica, social y económica que le es propia y específica al fenómeno social que se intentaestudiar. Se plantea entonces quesinese intento de comprensión y reflexión no es posible la explicación del individuo ni del grupo social, por lo que se hace o se asume como aspecto fundamental de 
la práctica etnográfica desde entonces, independientemente que ésta se haga fuera de un enfoque antropológico. Sin embargo, muchos de los aspectos relacionados al quehacer etnográfico parecen ser obviados, soslayados. Es decir, no son tomados en cuenta a la hora de ser utilizada la etnografía como soporte metodológico en algunas (cuando no en muchas) investigaciones que "intentan superar" el paradigma positivista.

En la actualidad, es común (por lo menos demasiado común en los trabajos e investigaciones de postgrado a los cuales me invitan a participar como asesor, evaluador, jurado, etc.) que se obvie dicha reflexión y se asume, incluso como principio, que cualquiera sea la postura epistemológica y metodológica, las mismas son válidas para cualquier tipo de problema. En otas palabras, el mismo principio positivista, pero a la inversa, que establece que cualquiera sea el problema, el enfoque, el tratamiento metodológico es, o debe ser el mismo. En este sentido, no es extraño encontrar "investigaciones" que aplicaron una supuesta estrategia metodológica cualitativa, o específicamente etnográfica, en donde no se establece una clara relación entre el "sujetos de estudio" y lo simbólico, fenomenológico, subjetivo, que subyace e incide en el mismo, es decir, lo sociocultural.

El llamado boom cualitativo ha puesto de moda lo que podría denominarse como una "hora loca metodológica", es decir, una especie de "anarquía" en cuanto a la rigurosidad metodológica, o en el caso que me atiende, una "locura etnográfica" (que no tiene nada que ver con el "desorden etnográfico" planteado por Marcus (2008), ya que entre otros aspectos supone, o existe la falsa creencia, de que la asunción de un modelo paradigmático distinto al positivista es algo innovador, insurgente y que le otorga a quien lo asume una especie de "patente" para hacer, sugerir o afirmar cualquier cosa sin ningún tipo de argumentos, así como para librarse de toda atadura u obligación acerca de realizar algún tipo de reflexión epistemológica referida a las razones por las cuales se asume o asumió una determinada estrategia metodológica, que no en pocos casos, catalogan como "estudio de tipo etnográfico" y de esta manera igualar al mismo como un "estudio cualitativo". 
Es por esto que, aunque desde un enfoque antropológico la utilización del método etnográfico ya no precisa de grandes explicaciones y/o justificaciones, ya que éste es su método, considero que fuera de dicho enfoque no solo debe justificarse su conveniencia, su definición y entendimiento pleno, sino que, además, debe demostrarse su utilidad y validez, y son precisamente estos aspectos los que generalmente suelen estar ausentes en la mayoría de este tipo de estudios que pretenden ser etnográficos.

La ausencia de una mínima reflexión acerca de la conveniencia de la utilización de un determinado método, entre otras carencias, deja como consecuencia que no se pueda establecer con claridad cuáles fueron las razones para la utilización del mismo, así como lo que implica su utilización o cómo fue su posterior desarrollo. Esta forma de usos de las llamadas "metodologías cualitativas" y particularmente de la etnografía como método, fuera de un enfoque antropológico, tiende a banalizar y/o vulgarizar a las mismas, concretándolo o reduciéndolo a una práctica insustancial y muchas veces sin sentido cuando se pretende que por el solo hecho de utilizar y aplicar algunas "entrevistas abiertas" junto a unas supuestas "observaciones participantes", se está utilizando una "metodología cualitativa" o peor, que se está haciendo etnografía.

Precisamente Marcus (2008) hace una crítica significativa al respecto al señalar que de la "experimentalidad" que desarrolló la etnografía y que la hizo poderosa en su afán de una mejor comprensión de lo cultural se ha llegado a una situación de "estado incierto" de la misma.

Esta situación repercute enormemente en la calidad de los resultados en cuanto a producción de conocimiento, trayendo como consecuencia, descripciones que no son tales o muy deficientes, así como la redacción de informes cargados de declaraciones y afirmaciones (en formas de supuestas e incomprensibles categorías y enunciados) que no tienen ningún tipo de referente histórico-social y mucho menos empírico, dando paso y/o incurriendo en muchos casos, bajo el argumento de un supuesto afán "innovador", en lo que J. Padrón (2006) bien ha definido como "cantinflerísmo académico". 


\section{Acerca de los inicios y el desarrollo de la etnografía}

No es mi intención hacer una exposición detallada acerca del desarrollo histórico de la etnografía, para ello recomiendo leer a Gómez Pellón (2017) y Guber, R. (2001) por citar algunos que creo son unos muy buenos trabajos al respecto. Mucho menos pretendo hacer un "manual" (de los cuales hay muchos y no muy buenos a mi gusto) para indicar cómo se hace etnografía. Soy de los que creen que más que un simple método, la etnografía es un proceso metodológico integral que incluso aún hoy se encuentra en desarrollo y que solo se aprende a hacerla, haciéndola, pero tomando en cuenta o conociendo sus principios básicos. De tal manera, y con base a la experiencia propia, considero que buena parte de la comprensión de la etnografía como proceso metodológico pasa por comprender algunos aspectos centrales del desarrollo histórico de la misma.

Primeramente, debo decir que tal y como se conoce, y suele hacerse hoy, la etnografía tiene una fuerte influencia norteamericana, precisamente por Boas y por la llamada escuela de Chicago, a través de las proposiciones y trabajos de Robert Park fundamentalmente, quienes a su vez recibieron una fuerte influencia de la antropología británica de finales del Siglo XIX y principios del XX (Calderón, 2013).

La mayoría de los pensadores y epistemólogos de la etnografía como método, concuerdan en establecer que fue el pensamiento darwinista y/o el evolucionismo biológico aplicado a lo social desde el siglo XVIII, con su marcado componente empírico, lo que le dio el suficiente impulso a la articulación de la etnografía como proceso confiable y útil, sobre todo para la recolección y procesamiento de información e interpretación de los nuevos grupos socioculturales contactados, específicamente para facilitarle a los centros de poder (básicamente europeos) la comprensión de los grupos colonizados. Precisamente a este contexto histórico de subyugación colonial se relacionará a la etnografía con las ideas de "estudio de gentes atrasadas", "primitivas", "salvajes", etc.

Sin embargo, el enfoque epistemológico bajo el cual se desarrolla el método etnográfico surge incluso mucho antes con las reflexiones 
que se hacen sobre las primeras expediciones organizadas de manera sistemática a lo largo de la historia en todo el mundo que incluyen los escritos de Herodoto, Marco Polo, Ibn Battuta, entre otros, y que lentamente condujeron a la conformación o producción de un tipo específico de conocimiento, que en el caso de la etnografía se refiere al conocimiento de los valores, patrones, características, es decir, de las formas de Ser, Pensar y Actuar, de los distintos grupos humanos.

Es así como, por ejemplo, del replanteamiento y reconfiguración geográfica que se desarrolla sobre todo a partir del siglo XIV, se pasó a la observación y registro (medianamente estandarizado) de las prácticas y de los hechos humanos relacionados a las formas de vida y costumbres de quienes poblaban los nuevos territorios. Es decir, se procuró un tipo de descripción, precisamente, de ésos hechos, acciones, conductas, creencias, etc., de los "otros", de los "extraños" y "no civilizados" y que eran susceptibles de ser observados y registrados. Es este tipo de práctica expedicionaria la que servirá de sustento para la creación de la etnografía (primero como forma de recolección de información y luego como forma de interpretación de los registros) término que es utilizado solo a partir del siglo XIX, cuando se establecieron las primeras categorías de análisis de los hechos sociales (Camacho, 2000).

Otro hito importante y poco reconocido en la conformación de lo que hoy se concibe como el método etnográfico lo representó la expansión de las ideas marxistas en Europa. Con la influencia del marxismo y del materialismo histórico, en ese mismo siglo XIX, surgió un procedimiento que constituye una integración de hechos materiales y humanos que estructuró un conjunto de procesos a los que se les llamó Dialéctica, la cual permite una explicación de fondo acerca de las relaciones que subyacen en la misma acción socializada y sistemática que, por supuesto, va a permear la práctica y los escritos etnográficos posteriores.

Para el final del siglo XIX y la primera mitad del XX, las llamadas ciencias humanas y sociales se establecieron como tales, en buena medida gracias al desarrollo de la sociología, la psicología y la antropología, fundamentalmente. En cada una de éstas disciplinas, en mayor o menor grado, la estructuración conceptual de las mismas 
y sus metodologías específicas se verán fuertemente influenciadas por el evolucionismo y el positivismo en pos, entre otras consideraciones, de alcanzar el estatus científico. En el caso particular de la antropología será la unidad y diversidad del género humano la que ayudará a la definición de su objeto de estudio y la etnografía como su método particular.

En los inicios del siglo XX nació en Estados Unidos, como ya se apuntó antes, con el naturalista y matemático alemán Franz Boas (1858-1942), la idea de una antropología cultural. Se ha afirmado que la misma representa una especie de síntesis angloamericana de la antropología, ya que supone la fusión de dos tradiciones antropológicas originales (la etnología y la antropología física). Con la misma comienza a desarrollarse una idea principal en el seno de la etnografía: la observación in situ como eje fundamental de dicha práctica, fundamentalmente a partir de los trabajos de W. Malinowski (1883-1942) y de otros clásicos antropólogos, sobre todo de antropólogos de origen inglés. La práctica etnográfica de éstos últimos estableció la necesidad de un mejor conocimiento de lo sociocultural y forjó el requerimiento de liberar sus potencialidades del cerco evolucionista y positivista utilizando para ello las técnicas y procedimientos que les fueran necesarios en el campo.

Esto último, es importante destacarlo, a riesgo de que me tilden como "positivista": la tal "liberación" del encierro positivista no supuso, ni supone aún, obligatoria y necesariamente el abandono o la negación del referente objetivo y/o empírico con el cual nació la etnografía. De tal manera que resulta importante conocer (y una necedad negar) que la etnografía se haya fundado, construido y desarrollado bajo los principios empiristas / cuantitativos de la ciencia basados en los registros derivados de la observación y la experiencia (Soto Ramírez, 2009). Principios que sin constituirse en ejes centrales de la etnografía actual, suelen resultar muy disuasivos y esclarecedores en la exégesis de los fenómenos socioculturales cuando éstos pueden ser referentes.

Podemos decir, entonces, que la etnografía, o más bien la práctica etnográfica, tiene entre otras, unos orígenes y unos referentes históricos que son muy remotos, razón por la cual se puede afirmar 
que no es una práctica nueva. En ese sentido, la "novedad", el "esnobismo académico" al que algunos la intentan relacionar no tiene ningún asidero, por lo menos así lo establece su desarrollo histórico. Por otra parte, la íntima relación de la etnografía con el empirismo y el positivismo con la cual nació y se desarrolló son aspectos de la cual no tiene por qué avergonzarse y a la que mucho menos tiene necesariamente que renunciar.

\section{Del concepto y la práctica etnográfica y algunos otros aspectos de interés}

La etnografía es el método privilegiado por la ciencia antropológica para el desarrollo de estudios de orden socio-cultural. No obstante, si se indaga entre los hacedores de etnografía -que no solamente son los antropólogos- parece no existir un concepto enteramente unificado sobre lo que concretamente es el método etnográfico. En ese sentido, Hammerley y Atkinson (1993) señalan que:

Existe desacuerdo sobre si la característica distintiva de la etnografía es el registro del conocimiento cultural (Spradley \& McCurdy, 1980), la investigación detallada de padrones de interacción social (Gumperz, 1981) o el análisis holístico de sociedades (Lutz, 1981). Algunas veces la etnografía se define como esencialmente descriptiva, otras veces como una forma de registrar narrativas orales (Walker, 1981); como contraste, sólo ocasionalmente se pone el énfasis en el desarrollo y verificación de teorías (Glaser y Strauss, 1967)... (p. 1).

De tal manera que la etnografía muchas veces se define por su finalidad, otras por sus procedimientos y otras por lo que pudiera llegar a demostrar. Sin embargo, ello no supone un "anarquismo" procedimental y sin argumentos, y mucho menos una "orfandad" definitoria ya que pueden ser identificados aspectos fundamentales que le son propios y que en última instancia ayudan a definirla y enmarcan su proceder. 
En tal sentido, un aspecto importante para la comprensión del concepto de etnografía tiene que ver con la definición del concepto o la noción de cultura. E. Tylor (1871) fue uno de los primeros que configuró el término cultura como “...ese complejo total que incluye conocimiento, creencia, arte, moral, ley, costumbres y otras aptitudes y hábitos adquiridos por el hombre como miembro de la sociedad" (p. 64). A partir de la definición aportada por Tylor se empieza a configurar el objeto de estudio de la antropología, dentro de un movimiento que rechazaba las teorías evolucionistas que vinculaban o atribuían a la raza, a la herencia biológica, las diferencias de los distintos grupos humanos (Soto Ramírez, 2009).

En cuanto a la etnografía, como ya es harto conocido, etimológicamente el término traduce algo como: "escritura o descripción sobre o acerca de un pueblo o grupo de personas". Asumida así, dicha etimología encierra varias claves importantes, ya que es precisamente en torno a las ideas que se desprenden de esta definición que se ha construido buena parte de su conceptualización. Para ello basta con hacer una revisión de algunos conceptos de sus principales definidores.

Uno de los conceptos más "populares" y referidos sobre lo que es la etnografía, lo aporta Aguirre (1997), quien la define “... como el estudio descriptivo de la cultura de una comunidad o de alguno de sus aspectos fundamentales..." (p. 3). De este tipo de conceptualización, se deduce un significado que se corresponde en su totalidad con la etimología antes señalada. Sin embargo, a la etnografía también se le suele definir como mucho más que un simple método, ya que Wolcott (1975), entre otros, la han definido como una "ciencia" para la descripción de la cultura, en el entendido de que la considera algo más que un simple método.

Como quiera que sea, existen en este tipo de definiciones dos ideas o categorías destacadas acerca de lo que la etnografía hace y sobre qué lo hace. Es decir, describe (descripción) sobre (acerca de) la cultura. Estas características están presentes hasta en la corriente posmoderna cuando se define a la etnografía como “...primero y ante todo una propuesta descriptiva en la que el investigador intenta ser fiel 
a la hora de describir la naturaleza del discurso social de un grupo de personas..." (Geertz, 1987, p. 27).

Por otra parte, existen conceptos más amplios que apuntan a definir la etnografía como un "...proceso metodológico global que caracteriza a la antropología social...”. (Díaz de Rada \& Velazco, 1997, p. 69). En este caso, la idea de proceso supone la conjunción de una serie de acciones, más allá de la simple descripción de hechos, para la cristalización de la etnografía.

De igual manera, existen otras ideas que paralelamente se van a desprender de esas nociones básicas relacionadas a la "descripción" y a la "cultura" cuando a la etnografía la asocian "... al estudio de gentes distintas a nosotros y al uso de métodos diferentes a los que provee el diseño experimental y la medición cuantitativa" (Hymes, 1982, p. 176).

Este tipo de conceptualización supone que la etnografía solo es posible en un contexto donde lo que se pudiera considerar como el "objeto de estudio" es el otro, el "extraño" a nuestra forma de ser, pensar y actuar; el otro cultural. Esta idea admite también una antigua y superada "tradición" antropológica: la imposibilidad del uso de la etnografía para el autoestudio cultural. De la misma manera implica la ejecución de un trabajo de campo y en consecuencia el ejercicio de la observación rigurosa y la participación del etnógrafo con y en el entorno socio cultural estudiado.

Pero supone también otra imposibilidad, la de "objetivar" el hecho cultural. Este es un principio ontológico fundamental en el desarrollo de la etnografía como método. En ese sentido, varios autores, entre ellos Berger y Luckman (1986), por ejemplo, parten del supuesto básico de que la realidad sociocultural no es un hecho dado, sino que se construye en la vida cotidiana, en el compartir diario de los sujetos, y es en esa cotidianidad en donde se generan las particulares formas que permiten el conocimiento de los grupos.

Es decir, los sujetos (portadores de la cultura) son los únicos capaces de reflejar la realidad a través de sus prácticas discursivas, de su razonamiento, del significado que le dan a sus acciones cotidianas, por lo tanto, no se trataría (solamente) de establecer frecuencias o 
variables, ni de mediciones cuantitativas como lo establecen las premisas positivistas, ya que éstas suelen no aportar lo suficiente para la explicación de los significados de ciertos hechos, en tanto que la etnografía trata es de entender, de explicar los fenómenos sociales desde la perspectiva particular de dichos sujetos y cómo éstos fenómenos pueden ser relacionados con el contexto social e histórico donde se enmarcan. De alguna manera, es a esto también a lo que se refiere Clifford Geertz (1987), cuando asume:

...que el hombre es un animal inserto en tramas de significación que él mismo ha tejido... que el análisis de la cultura ha de ser por lo tanto, no una ciencia experimental en busca de leyes, sino una ciencia interpretativa en busca de significaciones (p. 20).

Esto es lo que le da el carácter particular de conocimiento, como un origen y una expresión social-práctica, que se hace evidente en los comportamientos y en las prácticas discursivas dentro de las relaciones sociales.

Es esta epistemología la que le otorga a la etnografía la necesaria flexibilidad para abordar los hechos socioculturales, ya no solamente del "otro extraño", sino también, como ciertamente establece Martínez (2005)“... de cualquier grupo humano que constituya una entidad cuyas relaciones estén reguladas por la costumbre o por ciertos derechos y obligaciones recíprocos" (p. 2). De hecho, en Antropología ya no se estudia de manera exclusiva al "otro exótico", sino que se estudia al otro que puede ser otro respecto a nosotros mismos, el otro social, cultural, económico, étnico, sexual, incluso al otro íntimo, pues la representación del individuo es una construcción social que le interesa ya no solo a la antropología, es también porque toda representación del individuo es necesariamente una representación del vínculo social que le es circunstancial (Calderón, 2013).

Como resumen de lo antes expuesto en este punto, se pueden señalar algunos aspectos que resaltan, en primer lugar: la etnografía se refiere, en cualquiera de sus acepciones y tendencias, al ejercicio descriptivo de lo cultural. Por otra parte, se puede afirmar que este ejercicio de descripción de lo cultural se fundamenta en principios 
onto/epistemológicos que liberan las potencialidades del método sin que ello suponga una renuncia a la rigurosidad metodológica. De tal manera que, no es posible la utilización del método etnográfico, ni la cristalización misma de la etnografía, fuera del ámbito de estudio de lo sociocultural y de cómo éste incide en el comportamiento de los individuos.

\section{Acerca de los supuestos y propósitos de la etnografía}

Desde el punto de vista ideológico, soy de los que comparte la idea de que la producción de conocimiento no es ni ha sido nunca un proceso absolutamente neutral. La investigación cualitativa ha asumido este principio que, bien entendido, se convierte en una de sus mayores fortalezas en tanto que no oculta sus intenciones. Sin embargo, de dicha fortaleza se tiende a abusar.

El valor intrínseco y primordial a la investigación es la producción de conocimiento, o lo que es lo mismo, la búsqueda de "la verdad", cuyos límites y fronteras son establecidos por la ética y la moral que rigen el pensamiento de los investigadores en el proceso de investigación. A partir de entonces cualquier otro valor puede ser agregado, de tal manera que, si la producción de conocimiento se enmarca, como suele ser, dentro de un modelo ideológico/político o es funcional al mismo, es otra la discusión, la cual va a depender de los argumentos que se presenten al respecto.

Si bien es cierto que pudiera decirse que el énfasis de la etnografía ha variado y seguirá variando con el tiempo en tanto sus distintas tendencias, sus fines y/o propósitos se han mantenido, los cuales han apuntado siempre a establecer, caracterizar, interpretar, comprender las conductas, patrones, valores de los grupos de personas, dentro de un entorno particular y un marco general, independientemente del marco político ideológico desde dónde se haga.

En tal sentido, Hymes (1982) sugiere algunos propósitos que debe plantearse toda investigación etnográfica que perfectamente pueden ser aplicados en cualquier contexto de estudio y desde cualquier enfoque disciplinar. Es decir, no se trata solamente de aplicar unas determinadas técnicas de recolección de información, 
de lo que se trata es de obtener un conocimiento específico utilizando para ello las técnicas y herramientas más adecuadas.

En este contexto, se considera que la etnografía debe tener un propósito de contraste, una búsqueda de información específica y una interpretación general, o lo que es lo mismo, la etnografía tiene una naturaleza contextual y holística a la vez, además de un marcado carácter reflexivo de una determinada información:

La etnografía es algo más que una técnica residual; es el nombre para designar un método esencial, que se produce cuando estos tres aspectos se dan conjuntamente (Hymes, 1982, p. 178).

En todo caso, la etnografía se apoya en la convicción de que las tradiciones, roles, valores y normas del ambiente social (la cultura) en que se vive se van internalizando poco a poco y generan regularidades que pueden explicar la conducta individual y de grupo en forma adecuada y que dichas normas o valores pueden tener una explicación, no solo en la base material y objetiva que los sustenta, sino también que pueden tener explicación en lo más recóndito de la cosmovisión particular del grupo donde se dan los fenómenos.

En efecto, los miembros de un grupo, comparten una estructura lógica o de razonamiento que, por lo general no es explícita y que en algunos casos no responde a una "lógica racional", pero que se manifiesta en todos los aspectos de su vida y para aproximarse a esas estructuras escondidas hacen falta más que algunas entrevistas u observaciones.

Al respecto, cualquier técnica y/o procedimiento es válido en el "levantamiento" de la información etnográfica, desde la diagramación de mapas espaciales y mentales, líneas de tiempo, fuentes o levantamientos estadísticos, conversaciones y participación en actividades de interés, historias de vida, hasta registros gráficos, fílmicos, redes sociales, etc., siempre y cuando logren recoger lo más honesta y fidedignamente la información que se requiere y se apunte a los fines indicados. Efectivamente, la historia acerca de la producción del conocimiento científico nos remite al hecho cierto que los investigadores: 
...han asumido desde siempre una posición epistemológica que posteriormente se relaciona con los esquemas operativos pertinentes a la misma, con el propósito de orientar la investigación hacia la búsqueda de respuestas con métodos y técnicas idóneas para ese fin. (Camacho, 2000, p. 110-111).

Otro de los aspectos fundamentales, en mi criterio, en tanto la necesidad de comprender el discurso social desde la perspectiva particular de los sujetos, tiene que ver con la preparación del etnógrafo (que forma parte del proceso etnográfico) relacionado al conocimiento social e histórico del grupo que se pretende abordar. Al respecto Hymes (1982) establece que: "Cuanto más sabe el etnógrafo en el momento de entrar en el campo, más probable es que el resultado sea mejor" (p. 181). De tal manera que la reconstrucción o la aproximación étno-histórica mediante la revisión de archivos y toda la bibliografía y hemerografía posible y disponible, e incluso, indagar acerca de cómo se representan el pasado los sujetos de investigación, juega un papel importante en dicho proceso.

En atención a la mayoría de estos supuestos y propósitos, en mayor o menor medida, se han desarrollado las distintas tradiciones etnográficas, o por lo menos las más conocidas, a saber: la "etnografía clásica" o "funcional-estructuralista"; la "nueva etnografía" o "etnografía sistemática"; la "etnografía hermenéutica" o la llamada "etnografía crítica". Por otra parte, es por este tipo de características que a la etnografía suelen darle tres tipos de acepción: como enfoque, como método propiamente y como producto (Guber, 2001).

\section{Resumiendo}

Una cosa es hacer etnografía bajo el entendimiento de algunos aspectos relativos a su historia, a sus concepciones y a sus fines, y otra es utilizar simplemente algunas herramientas y técnicas que son utilizados para ello.

Sin embargo, no se trata de que cuando los docentes, administradores, médicos, etc., hagan etnografía tengan que hacer 
antropología, no. De lo que se trata es que para usar la etnografía se hace necesario e imprescindible que exista una relación directa entre el problema o tópico que se estudia y los aspectos socioculturales donde se inserta. De no existir esta relación, o de no establecerla, la etnografía como enfoque, método o producto no es útil, ni es posible.

Es decir, ciertamente cualquier problema de orden social (unos más que otros) puede ser abordado desde un enfoque, desde una perspectiva etnográfica siempre y cuando se sospeche o se establezca que existe una relación entre dicho problema y la cultura. Por ello, desde el punto de vista epistemológico, la etnografía es una interpretación que busca perennemente aproximarse a las formas y maneras de pensar, actuar, decir de un grupo sociocultural determinado.

Ahora bien, considero que, entre los variados aspectos que involucran la realización de una investigación relacionada o que intente abordar aspectos de orden sociocultural, y sobre todo si intenta "superar" el modelo positivista, debería o debe existir la necesidad, incluso antes del inicio de la misma, de reflexionar sobre el problema específico que se abordará y cómo éste tiene (o no) una relación con la cultura en dónde se expresa y si la misma juega un papel de incidencia significativa en el fenómeno social de interés. Creo que ésta es una de las formas en la que el investigador podrá asirse a algunas certezas sobre cuál o cuáles pueden ser las mejores maneras de acercarse a su objeto/sujeto de estudio así como de prever las implicaciones teórico-metodológicas subsiguientes.

De tal manera que la etnografía es más que un mero acto descriptivo de un hecho cultural. La etnografía implica un proceso riguroso, reflexivo, comprensivo y fidedigno de dicho hecho. En este sentido, cuando bajo este entendimiento se asume a la etnografía, ésta se convierte en lo que pudiéramos denominar como una "estrategia metodológica total" (en el sentido de Gustavo Cerati) que busca la comprensión de los fenómenos sociales desde una perspectiva cultural, y todo ello va más allá de los marcos paradigmáticos que intentan cercarla. 
Así, a lo largo de los numerosos campos en que ha sido propuesta la etnografía, o a veces algo parecido a ella, podemos encontrar diferencias considerables en cuanto a las prescripciones y a la propia práctica, pero lo que no puede obviarse es su carácter descriptivo, reflexivo y comprensivo de hechos socioculturales. La etnografía no tiene mucho que ver con un paradigma determinado, sino más bien con el hecho inexorable de que trata sobre la influencia de la cultura humana en los fenómenos sociales.

\section{Referencias}

Aguirre, A. (1997). Etnografía. Metodología cualitativa en la investigación sociocultural. México: Alfaomega.

Berger, P. L., Luckmann, T., \& Zuleta, S. (1968). La construcción social de la realidad (Vol. 975). Buenos Aires: Amorrortu.

Boas, F. (1896). The Limitations of the Comparative Method of Anthropology. Science, 4(103), 901-908. Recuperado de http:// www.jstor.org/stable/1623004

Boas, F. (1911). The mind of primitive man: a course of lectures delivered before the Lowell institute and the National university of Mexico, 1910-1911. Boston: Macmillan.

Calderón, L. (2013). Representaciones de lo indígena en el proceso de reconstrucción de la identidad. Recuperado de http://tesis.luz. edu.ve/tde_arquivos/3/TDE-2013-11-13T09:56:01Z-4254/ Publico/calderon_osuna_lenin_rafael.pdf

Camacho, H. (2000). Enfoques Epistemológicos y Secuencias Operativas de Investigación. (Tesis Doctoral). Universidad Rafael Belloso Chacín, Maracaibo. Recuperado de http://padron.entretemas.com.ve/Tesistas/TesisHermelinda.pdf

Cerati, G. (1959-2014). Músico argentino.

Díaz de Rada, A., \& Velazco, H. (1997). La Lógica de la Investigación Etnográfica. Madrid: Trotta.

Díaz de Rada, A., Velazco, H., \& García, F. (1993). Lecturas de Antropología para Educadores. El ámbito de la antropología de la educación y de etnografía escolar. Madrid: Trotta. 
Geertz, C. (1987) The Interpretation of Cultures. Nueva York: Basic Books.

Glaser, B., G. y Strauss, A., L. (1967) The Discovery of Grounded Theory: Strategies for Qualitative Research. Chicago: Aldine.

González, A. (1990). Etnografía y Comparación: La investigación intercultural en Antropología. Barcelona: Bellaterra.

Gumperz, J. (1981). Citado en: Hammersley, M., y Atkinson, P. Etnografía: métodos de investigación. Barcelona: Paidós.

Guber, R. (2001). La etnografía, método, campo y reflexividad. Bogotá: Grupo Editorial Norma.

Hamme, M., \& Atkinson, P. (1994). Etnografía Métodos de Investigación Ed. Paidós. Barcelona

Hymes, D. (1982). ¿Qué es la Etnografía? En: Velasco, H., García, J., \& Díaz de Rada, A. (Eds) Lecturas de Antropología para Educadores. Madrid: Trotta.

Kuhn, T. (1971). La Estructura de las Revoluciones Científicas. México: Fondo de Cultura Económica.

Marcus, G. (2008). El o los fines de la etnografía: del desorden de lo experimental al desorden de lo barroco. Revista de Antropología Social, 17 27-48.

Martínez Miguélez, M. (2005). El Método Etnográfico de Investigación. Recuperado de http://prof.usb.ve/miguelm/metodoetnografico.html

Lutz, F. W. (1981). Citado en: Hammersley, M., y Atkinson, P. Etnografía: métodos de investigación. Barcelona: Paidós.

Padrón, J. (2006). Notas acerca del cantinflerísmo académico. (1 ${ }^{a}$ Parte, versión borrador). Disponible en http://maestriaengerenciaestrategica.blogspot.com/2011/04/curso-etica-de-la-profesion.html

Pellón, E. G. (1995). La evolución del concepto de etnografía. En Etnografía: metodología cualitativa en la investigación sociocultural (pp. 21-48). Marcombo. Disponible en http://torrefdz. es/antropusi49.htm 
Peñaranda, F. (2004). Consideraciones epistemológicas de una opción hermenéutica para la etnografía. Revista Latinoamericana de Ciencias Sociales, Niñez y Juventud, 2 (2), 167-189.

Spradley, J. P., \& McCurdy, D. W. (1980). Anthropology, the cultural perspective. New York: John Wiley \& Sons.

Soto Ramírez, J. (2009). Reflexiones antropológicas sobre la unidad, la diversidad y la cultura. Culturales, 5(10), 91-120.

Tylor, E. (1871). Cultura Primitiva. En: Bohannan, P. y Glazer, M. Antropología, lecturas. España: Mc. Graw-Hill.

Walker (1981). Citado en: Hammersley, M., y Atkinson, P. Etnografía: métodos de investigación. Barcelona: Paidós.

Wolcott, H. (1975). Criteria for an ethnographic approach to research in schools. Human organization, 34(2), 111-127. 